\title{
MicroRNA-424 alleviates neurocyte injury by targeting PDCD4 in a cellular model of cerebral ischemic stroke
}

\author{
HOU-WEI REN ${ }^{1}$, BIN GU ${ }^{1}$, YUE-ZHAN ZHANG ${ }^{2}$, TING GUO ${ }^{1}$, \\ QIAN WANG ${ }^{1}$, YUE-QIN SHEN ${ }^{1}$ and JUN WANG $^{1}$ \\ ${ }^{1}$ Department of Emergency, Taizhou People's Hospital Affiliated to Nantong University, Taizhou, Jiangsu 225300; \\ ${ }^{2}$ Department of Emergency, The Fourth Affiliated Hospital of Zhejiang University, \\ School of Medicine, Yiwu, Zhejiang 322000, P.R. China
}

Received September 22, 2019; Accepted August 26, 2021

DOI: $10.3892 /$ etm.2021.10888

\begin{abstract}
Cerebral ischemic stroke is the primary cause of stroke-associated mortality and disability, and current therapeutic options are limited and ineffective. The present study aimed to investigate the potential of apoptotic therapy and the role of microRNA (miR)-424 in cerebral ischemic stroke. PC12 cells, a cloned cell line from rat adrenal pheochromocytoma, were treated with $\mathrm{CoCl}_{2}$ to construct a cellular ischemia model. mRNA and protein levels of programmed cell death protein 4 (PDCD4), Bcl-2, Bax, caspase-3, PI3K and AKT were evaluated using reverse transcription-quantitative PCR and western blot analyses, respectively. Cell Counting Kit-8 assays were performed to examine cell viability in the ischemia model. Flow cytometry was conducted to evaluate the apoptosis of ischemic cells. Furthermore, a luciferase assay was performed to verify the target gene of miR-424. It was revealed that the expression level of miR-424 was downregulated in the ischemia model, while the expression of PDCD4 was upregulated. Moreover, the expression of miR-424 was increased after treatment with miR-424 mimics. The mRNA and protein expression of PDCD4 was upregulated after transfection with pcDNA3.1-PDCD4. PDCD4 was predicted and demonstrated to be a target of miR-424. Notably, overexpression of miR-424 increased cell viability and inhibited apoptosis in the ischemia model, which was reversed by co-treatment with pcDNA3.1-PDCD4. Furthermore, overexpression of miR-424 regulated the expression of PDCD4, Bax, Bcl-2, phosphorylated-PI3K/AKT and caspase-3, which was restored after co-transfection with pcDNA3.1-PDCD4. Collectively, the results indicated that miR-424 regulated the
\end{abstract}

Correspondence to: Dr Jun Wang, Department of Emergency, Taizhou People's Hospital Affiliated to Nantong University, 366 Taihu Road, Taizhou, Jiangsu 225300, P.R. China

E-mail: 15061008998@163.com

Key words: microRNA-424, cerebral ischemic stroke, apoptosis, programmed cell death protein 4 progression of cerebral ischemic stroke in a cellular model by targeting PDCD4.

\section{Introduction}

Stroke is characterized with high morbidity, mortality and disability (1). Strokes can be stratified into ischemic and hemorrhagic categories, which account for $~ 80 \%$ and $20 \%$ of all strokes, respectively (2). Cerebral ischemic stroke is induced by the development of a thrombus within the major cerebral artery, contributing to the apoptosis of neuronal cells $(3,4)$. The only efficacious therapies for cerebral ischemic stroke are dissolving the thrombus or using tissue plasminogen activators (5). However, the number of patients with cerebral ischemic stroke receiving these treatments is $\leq 4.5 \%$ (6). Additionally, secondary neuronal death may occur due to cerebral infarction, resulting in long-term neurological deficits (7). The incidence and mortality rate of ischemic stroke has been increasing annually in China (8), exerting a notable threat to public health. In 2017, a survey of 155 urban and rural centers in 31 provinces in China showed that the age-standardized prevalence, incidence and mortality rates were $1,114.8$ per $100,000,246.8$ and 114.8 per 100,000 person-years, respectively (9). Therefore, there is a requirement to explore novel and effective treatment options for cerebral ischemic stroke. It has been reported that neuronal cell apoptosis following cerebral ischemia/reperfusion (I/R) is the primary cause of brain injury $(10,11)$. Prevention of neuronal cell apoptosis is a potential treatment aim for ischemic stroke (12). Therefore, it is important to explore the underlying mechanisms of ischemic neuronal death.

MicroRNAs (miRNAs/miRs) are a family of small single-stranded non-coding RNAs (13). miRNAs serve important roles in various cellular processes, including cell proliferation, differentiation and apoptosis (14). Dysregulation of miRNAs influences various diseases, including stroke (15). Various studies have reported that aberrant expression of miRNAs influences cerebral ischemic stroke. For example, upregulation of miR-130b mediates cerebral ischemic injury in astrocytes (16). Furthermore, upregulation of miR-145 alleviates primary astrocyte injury following cerebral ischemic stroke in vitro (17). Notably, the abnormal expression 
of miRNAs is associated with neurological disorders and regulates the expression of apoptosis-associated genes (18). Dysregulated expression of miR-525-5p ameliorates I/R injury-induced neuronal cell death in a study in adult rats (19). Upregulation of miR-9 has been reported to suppress neuronal apoptosis in rats with ischemic stroke (20). A previous study reported that lower levels of circulating miR-424 in patients with acute cerebral infarction may predict poor prognosis, where the expression of circulating miR-424 is negatively associated with the serum levels of certain pro-inflammatory cytokines (21). This suggests that circulating miR-424 may have the potential to serve as a prognostic marker and therapeutic target for the management of acute cerebral infarction. However, the potential mechanisms of miR-424 in cerebral infarction have not been fully elucidated.

The PC12 cell line is a cloned cell line from rat adrenal pheochromocytoma that is widely used as a model to study neuronal cell differentiation and function $(22,23)$. This cell line is favored due to its high stability, homogeneity and differentiation $(24,25)$. Hypoxia of brain tissue after cerebral infarction causes a large amount of hypoxia inducible factor (HIF)-1 accumulation, mitochondrial dysfunction, free radical eruption, which leads to nerve cell damage (26). Chronic hypoxia occurs in nerve cells due to the inability to efficiently transport and utilize oxygen after $\mathrm{CoCl}_{2}$ treatment (27). $\mathrm{CoCl}_{2}$ induced a series of changes in nerve cells similar to the effects of hypoxia (28). Therefore, $\mathrm{CoCl}_{2}$ is an ideal compound for establishing a chronic hypoxia model.

Programmed cell death factor 4 (PDCD4) is originally described as a tumor suppressor gene that exerts antineoplastic effects by promoting apoptosis (29). The present findings suggested that miR-424 may represent a promising biomarker for cerebral ischemic stroke. However, the mechanism of miR-424 in acute cerebral infarction remains unclear. In the present study, the regulatory role of miR-424 was investigated in an in vitro model of acute cerebral infarction.

\section{Materials and methods}

Cell culture. PC12 cells were obtained from American Type Culture Collection. Cells were incubated at $37^{\circ} \mathrm{C}$ in DMEM containing 5\% FBS, $100 \mathrm{U} / \mathrm{ml}$ penicillin and $100 \mu \mathrm{g} / \mathrm{ml}$ streptomycin (all from Gibco; Thermo Fisher Scientific, Inc.) at $5 \% \mathrm{CO}_{2}$ until they reached $60-70 \%$ confluence. The cells were then incubated either in medium supplemented with $200 \mu \mathrm{M}$ $\mathrm{CoCl}_{2}$ (cat. no. 618519; Sigma-Aldrich; Merck KGaA) or in normal medium. After $24 \mathrm{~h}$, cells were collected for subsequent experiments.

Transfection. miR-424-5p mimic (sense, 5'-CAGCAGCAA UUCAUGUUUUGAA-3' and antisense, 5'-CAAAACAUG AAUUGCUGCUGUU-3'). mimic negative control (NC; sense, 5'-UUCUCCGAACGUGUCACGUTT-3' and antisense, 5'-ACGUGACACGUUCGGAGAATT-3') were purchased from Shanghai GenePharma Co., Ltd. PC12 Cells were cultured $\left(8 \times 10^{5}\right.$ cells/well $)$ in 12 -well plates. After cells reached $70-80 \%$ confluence, they were transfected with $100 \mathrm{nM}$ miR-424 mimics or miR-negative control (NC) mimics (Shanghai GenePharma Co., Ltd.) using Lipofectamine ${ }^{\circledR} 2000$ (Invitrogen; Thermo Fisher Scientific, Inc.). For programmed cell death protein 4 (PDCD4) transfection, cells were treated with $1 \mu \mathrm{g} \mathrm{pcDNA3.1-PDCD4}$ or pcDNA3.1 (Addgene, Inc.) for $48 \mathrm{~h}$ using Lipofectamine 2000 according to manufacturer's instructions. After transfection, cells were subjected to ischemic treatment for $12 \mathrm{~h}$.

Cell Counting Kit-8 (CCK-8). At 0, 12, 24 and $48 \mathrm{~h}$ post-transfection, cells were re-seeded in a 96 -well plate $\left(5 \times 10^{3}\right.$ cells/well). Each well was supplemented with $10 \mu \mathrm{l}$ CCK-8 solution (Dojindo Molecular Technologies, Inc.) and incubated at $37^{\circ} \mathrm{C}$ for another $2 \mathrm{~h}$ according to the manufacturer's protocol. The absorbance value at $450 \mathrm{~nm}$ was determined (Bio-Rad Laboratories, Inc.).

Flow cytometry. Cells were harvested and washed with pre-cooled PBS. Then cells were resuspended in $200 \mu \mathrm{l}$ binding buffer. Subsequently, cells were stained with FITC-Annexin V and propidium iodide (PI; BD Biosciences) in the dark for $20 \mathrm{~min}$ at room temperature. Subsequently, the data were evaluated with a BD FACSCalibur ${ }^{\mathrm{TM}}$ flow cytometer (Beckman Coulter, Inc.) using FlowJo software (version 10; FlowJo LLC). Sums of percentages of cells in early and late apoptosis were added as the total apoptosis ratio from each dot plot.

Reverse transcription-quantitative PCR (RT-qPCR). Total RNA from cells was isolated with TRIzol ${ }^{\circledR}$ reagent (Invitrogen; Thermo Fisher Scientific, Inc.) and the concentration of total RNA was determined with NanoDrop ${ }^{\mathrm{TM}} 1000$ (NanoDrop Technologies; Thermo Fisher Scientific, Inc.). A total of $1 \mu \mathrm{g}$ RNA was reversely transcribed using a TaqMan MicroRNA Reverse Transcription kit (Applied Biosystems; Thermo Fisher Scientific, Inc.), The reverse transcription were conducted under the following conditions: $42^{\circ} \mathrm{C}$ for $60 \mathrm{~min}, 72^{\circ} \mathrm{C}$ for 15 min. Amplification of cDNA was conducted using a RAPA3G SYBR Green qPCR Mix (cat. no. A2250A; HaiGene Biotech Co., Ltd.; https://www.haigene.cn/html/show-226. html). The PCR reactions were conducted under the following conditions: $95^{\circ} \mathrm{C}$ for $10 \mathrm{~min}$, followed by 35 cycles of $94^{\circ} \mathrm{C}$ for $30 \mathrm{sec}, 58^{\circ} \mathrm{C}$ for $30 \mathrm{sec}$ and $72^{\circ} \mathrm{C}$ for $35 \mathrm{sec}$, with a final extension at $72^{\circ} \mathrm{C}$ for $10 \mathrm{~min}$. U6 and GAPDH were used to normalize miRNA and mRNA, respectively. The sequences of the primers used for qPCR were as follows: miR- 424 forward, 5'-TTCAAAACATGAATTGCTGCTG-3' and reverse, 5'-CT CAACTGGTGTCGTGGA-3'; U6 forward, 5'-GCTTCGGC AGCACATATACTAAAAT-3' and reverse, 5'-CGCTTCACG AATTTGCGTGTCAT-3'; PDCD4 forward, 5'-TCCCTA ATTCTCCATGGTGCT-3' and reverse, 5'-CGCCTTTTT GCCTTGGCATT-3'; Bax forward, 5'-TGAAGGACGCAC GTTCAG-3' and reverse, 5'-CCTGCTCGATCCTGGATG AAA-3'; Bcl-2 forward, 5'-CTTTGAGTTCGGTGGGG TCA-3' and reverse, 5'-GGGCCGTACAGTTCCACAAA-3'; caspase-3 forward, 5'-GGAACCATCATACATGGAAGCG-3' and reverse, 5'-TTCCCTGAGGTTTGCTGCAT-3'; GAPDH forward, 5'-GGAGCGAGATCCCTCCAAAAT-3' and reverse, 5'-GGCTGTTGTCATACTTCTCATGG-3'. mRNA expression was determined using the $2^{-\Delta \Delta \mathrm{Cq}}$ method (30). Each experiment was performed in triplicate.

Western blotting. Total protein was extracted from PC12 cells using pre-cold RIPA buffer (Beyotime Institute of 
A

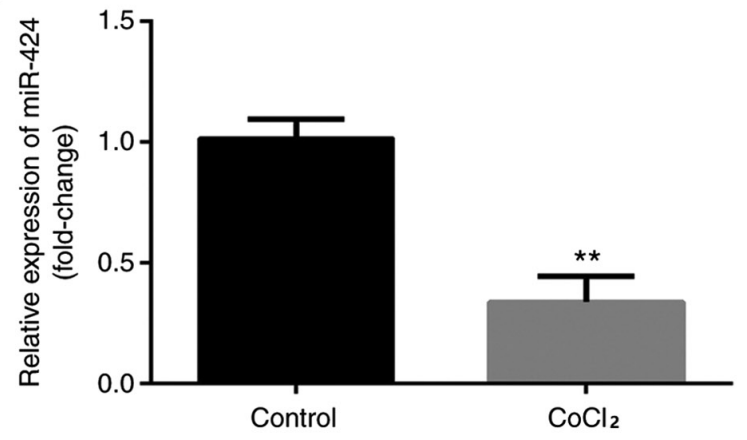

B

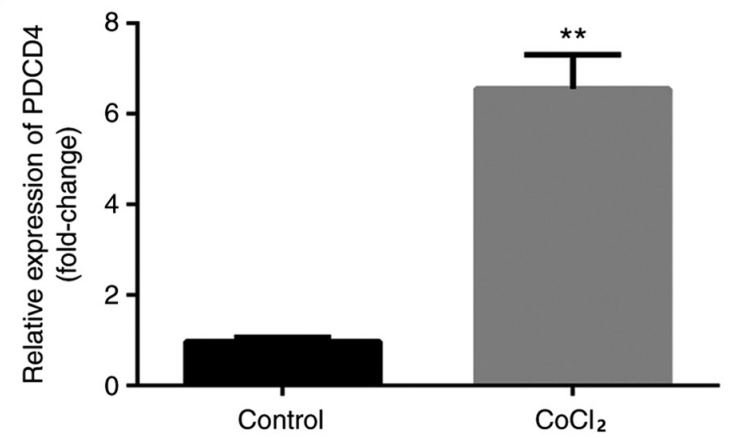

Figure 1. Expression of miR-424 and PDCD4 in a neuronal cell model of ischemia. (A) Expression of miR-424 was significantly decreased after treatment with $\mathrm{CoCl}_{2}$. (B) Expression of PDCD4 was significantly higher after treatment with $\mathrm{CoCl}_{2}$. ${ }^{* *} \mathrm{P}<0.01$ vs. Control. miR, microRNA; PDCD4, programmed cell death protein 4.

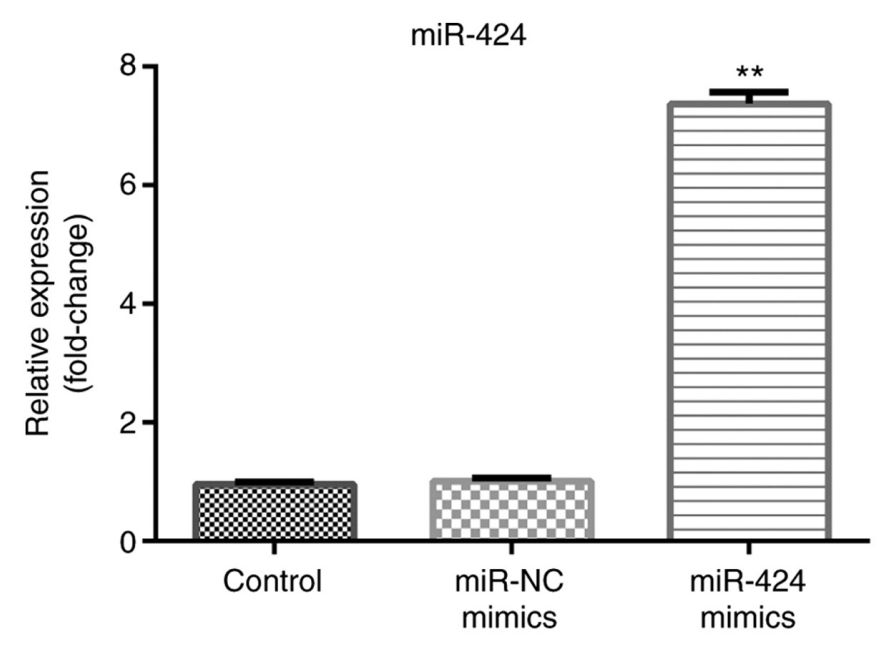

Figure 2. Expression of miR-424 following transfection with mimics. Expression of miR-424 was significantly increased after treatment with miR-424 mimics, while there was no significant difference between the control and miR-NC mimics groups. ${ }^{* *} \mathrm{P}<0.01$ vs. miR-NC mimics. miR, microRNA; NC, negative control.

Biotechnology) including protease inhibitor. Total protein was quantified using a bicinchoninic acid kit (Sigma-Aldrich; Merck KGaA). Proteins $(40 \mu \mathrm{g})$ were then separated via $10 \%$ SDS-PAGE, proteins were transferred onto PVDF membranes. The membranes were then blocked with $5 \%$ skimmed milk at room temperature for $1 \mathrm{~h}$. The membranes were incubated overnight at $4^{\circ} \mathrm{C}$ with anti-PDCD4 (1:1,000; cat. no. ab80590; Abcam), PI3K (1:1,000; cat. no. ab32089; Abcam), p-PI3K p85 (1:1,000; cat. no. ab278545; Abcam), AKT (1:1,000; cat. no. ab8805; Abcam), p-AKT (1:1,000; cat. no. ab81283; Abcam), Bax (1:1,000; cat. no. ab32503; Abcam), caspase-3 (1:1,000; cat. no. ab13847; Abcam) and GAPDH (1:1,000; cat. no. ab9485; Abcam) primary antibodies in the absence of light. Then cells were incubated with HRP-labeled anti-rabbit IgG secondary antibody (1:4,000; cat. no. ab6721; Abcam) or anti-mouse IgG secondary antibody (1:5,000, cat. no. ab6789, Abcam) for $2 \mathrm{~h}$ at room temperature. The bands were imaged with an Pierce ${ }^{\mathrm{TM}}$ ECL Western Blotting Substrate kit (cat. no. 32109; Thermo Fisher Scientific, Inc.) and band density was quantified using Quantity One v.6.2 software (Bio-Rad Laboratories, Inc.).

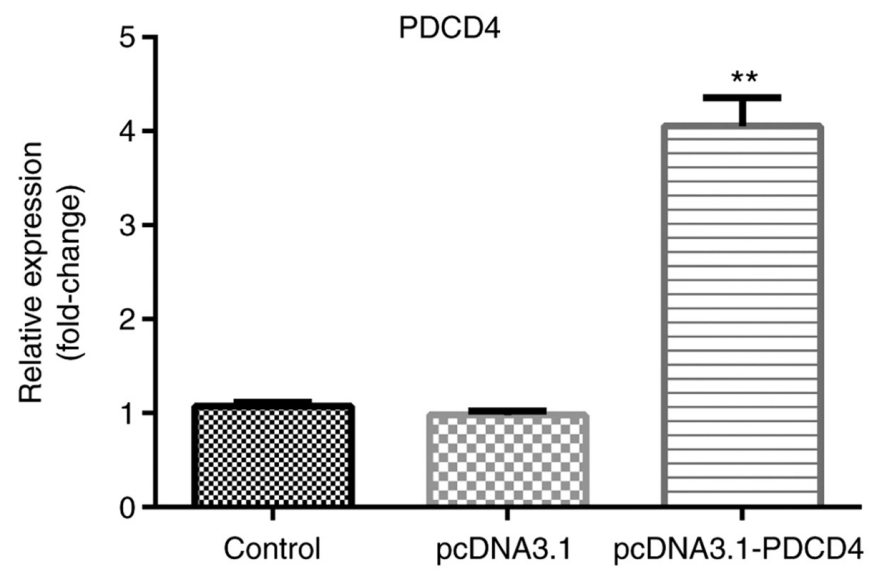

Figure 3. Expression of PDCD4 following transfection with overexpression vector. Expression of PDCD4 was significantly upregulated after transfection with pcDNA3.1-PDCD4. The difference between the control and pcDNA3.1 groups was not significant. ${ }^{* *} \mathrm{P}<0.01$ vs. pcDNA3.1. PDCD4, programmed cell death protein 4.

Dual-luciferase reporter assay. Bioinformatics analysis predicted using TargetScan 7.2 (http://www.targetscan. org/vert_71/) revealed that PDCD4 was a target of miR-424. For analysis of the predicted miRNA binding site, GCUGCUAA, located at the c*392-c*399 site of PDCD4 (NM_145341.4), a dual-luciferase reporter assay was performed to determine whether miR-424 directly targeted PDCD4. Complementary 50-60-bp DNA oligonucleotides consisting of the test sequence flanked by single-stranded overhangs encoding restriction enzyme sites SacI and HindIII in the multiple cloning sites of the pMIR-REPORT Luciferase plasmid (Ambion; Thermo Fisher Scientific, Inc.) were designed and synthesized. Synthetic oligonucleotides (Thermo Fisher Scientific, Inc.) annealing and ligation into the plasmid were performed according to the manufacturer's protocols. The DNA oligos used were as follows: Wild-type sense, 5'-CTATTTAGGGGGTAAAGTTAAGCTGCTAAAACCC CATGTTGGCTGCTGCTA-3' and antisense, 5'-AGCTTA GCAGCAGCCAACATGGGGTTTTAGCAGCTTAACTTT ACCCCCTAAATAGAGCT-3' and mutant sense, 5'-CTATTT AGGGGGTAAAGTTAACAGAAGAAAACCCCATGTTG GCT GCTGCTA-3' and antisense, 5'-AGCTTAGCAGC 
A

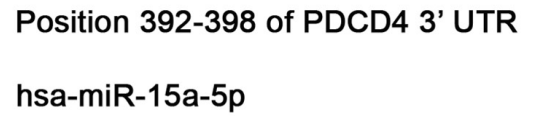

Mutant of PDCD4 3' UTR

\section{5' ...AGGGgGUAAAGUUAAGCUGCUAA... II II I I \\ 3' AAGUUUUGUACUUAACGACGAC \\ 5' ...AGgGgGUAAAGUUAACAGAAGAA..}

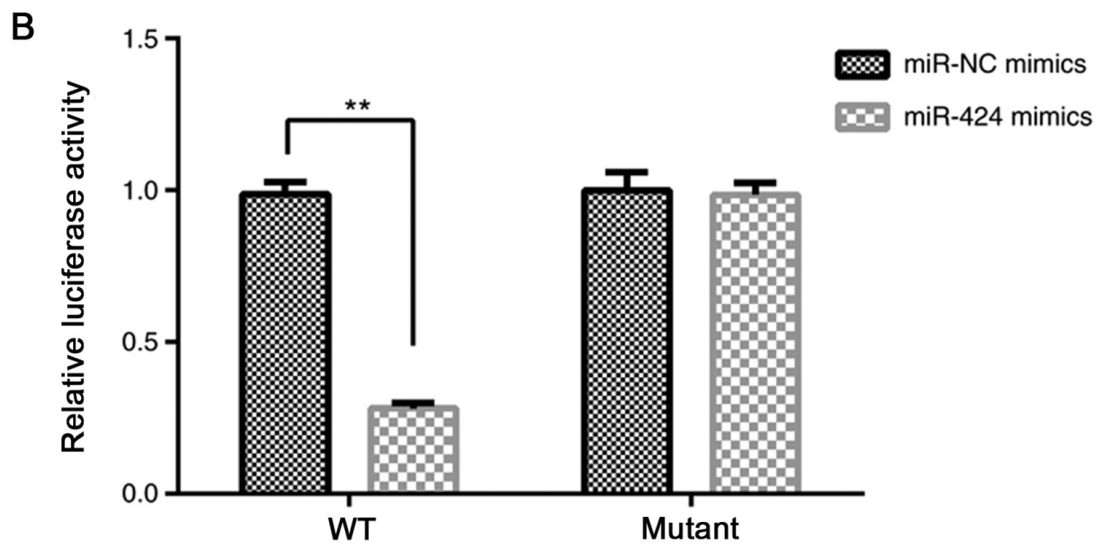

Figure 4. PDCD4 is a target of miR-424. (A) Sequence alignment revealed a binding site for miR-424 in the 3'UTR of PDCD4. (B) Luciferase activity of cells was significantly decreased after co-transfection with miR-424 mimics and PDCD4-3'UTR-WT, while there was no significant difference in the mutant groups. ${ }^{* *} \mathrm{P}<0.01$. miR, microRNA; PDCD4, programmed cell death protein 4; NC, negative control; UTR, untranslated region; WT, wild-type.

AGCCAACATGGGGTTTTCTTCTGTTAACTTTACCC CCTAAATAGAGCT-3'. PC12 cells were cultured $\left(8 \times 10^{4}\right.$ cells $\left./ \mathrm{cm}^{2}\right)$ into 12 -well plates. After the cells reached $70-80 \%$ confluence, $\mathrm{PC} 12$ cells were co-transfected with $100 \mathrm{ng}$ firefly luciferase constructs and $100 \mathrm{nM}$ miR-424 mimic or miR-NC mimic using Lipofectamine ${ }^{\circledR} 2000$ (Invitrogen; Thermo Fisher Scientific, Inc.). Renilla luciferase was used as the internal control for normalization. The luciferase activity was calculated using a dual-luciferase reporter assay (Promega Corporation) at $48 \mathrm{~h}$ post-transfection.

Statistical analysis. All experiments were repeated $\geq$ three times. Statistical analyses were performed using SPSS software (version 17.0; SPSS, Inc.). Data are presented as the mean \pm SD. Comparisons between two groups were performed using an unpaired Student's t-test. Comparisons of $>2$ groups were performed using one-way ANOVA followed by Tukey's post hoc test. $\mathrm{P}<0.05$ was considered to indicate a statistically significant difference.

\section{Results}

Expression of miR-424 and PDCD4 in ischemia model. RT-qPCR was performed to examine the RNA expression of miR-424 and PDCD4. Compared with normal PC12 cells, the expression of miR-424 was significantly decreased following $\mathrm{CoCl}_{2}$ treatment, whereas the expression of PDCD4 was significantly upregulated (Fig. 1A and B).

Transfection of miR-424 mimics in PC12 cells. Cells were transfected with miR-NC mimics or miR-424 mimics. The expression of miR-424 was significantly increased after the treatment with miR-424 mimics compared with miR-NC mimics, while there were no significant differences between the non-transfected control group and the miR-NC mimics group (Fig. 2).

Transfection of PDCD4 in PC12 cells. Cells were transfected with pcDNA3.1-PDCD4 or pcDNA3.1. As presented in Fig. 3, the expression level of PDCD4 in cells transfected with pcDNA3.1-PDCD4 was significantly upregulated compared with cells transfected with pcDNA3.1. There was no significant difference in expression between the pcDNA3.1 group and the non-treated control group.

PDCD4 is a target of $m i R-424$. PDCD4 was predicted as a target gene of miR-424 using TargetScan 7.2 (Fig. 4A). The results of the luciferase assay demonstrated that for the PDCD4-3'UTR-WT transfected cells, the luciferase activity was significantly decreased after transfection with miR-424 mimics compared with that in the miR-NC mimics group, whereas for the PDCD4-3'UTR-mutant transfected cells, transfection with miR-424 mimics exerted no significant effects on the luciferase activity (Fig. 4B).

Overexpression of miR-424 inhibits the apoptosis of ischemia-exposed cells. The apoptosis of ischemic PC12 cells was examined via flow cytometry. As presented in Fig. 5A and B, there was no significant difference between the control and NC group; however, the apoptosis rate significantly decreased after transfection with miR-424 mimics, which was reversed by co-transfection with pcDNA3.1-PDCD4.

Overexpression of miR-424 promotes the viability of ischemia-exposed cells. The viability of ischemic PC12 cells was determined using a CCK-8 assay. Overexpression of miR-424 significantly increased the viability of ischemic 

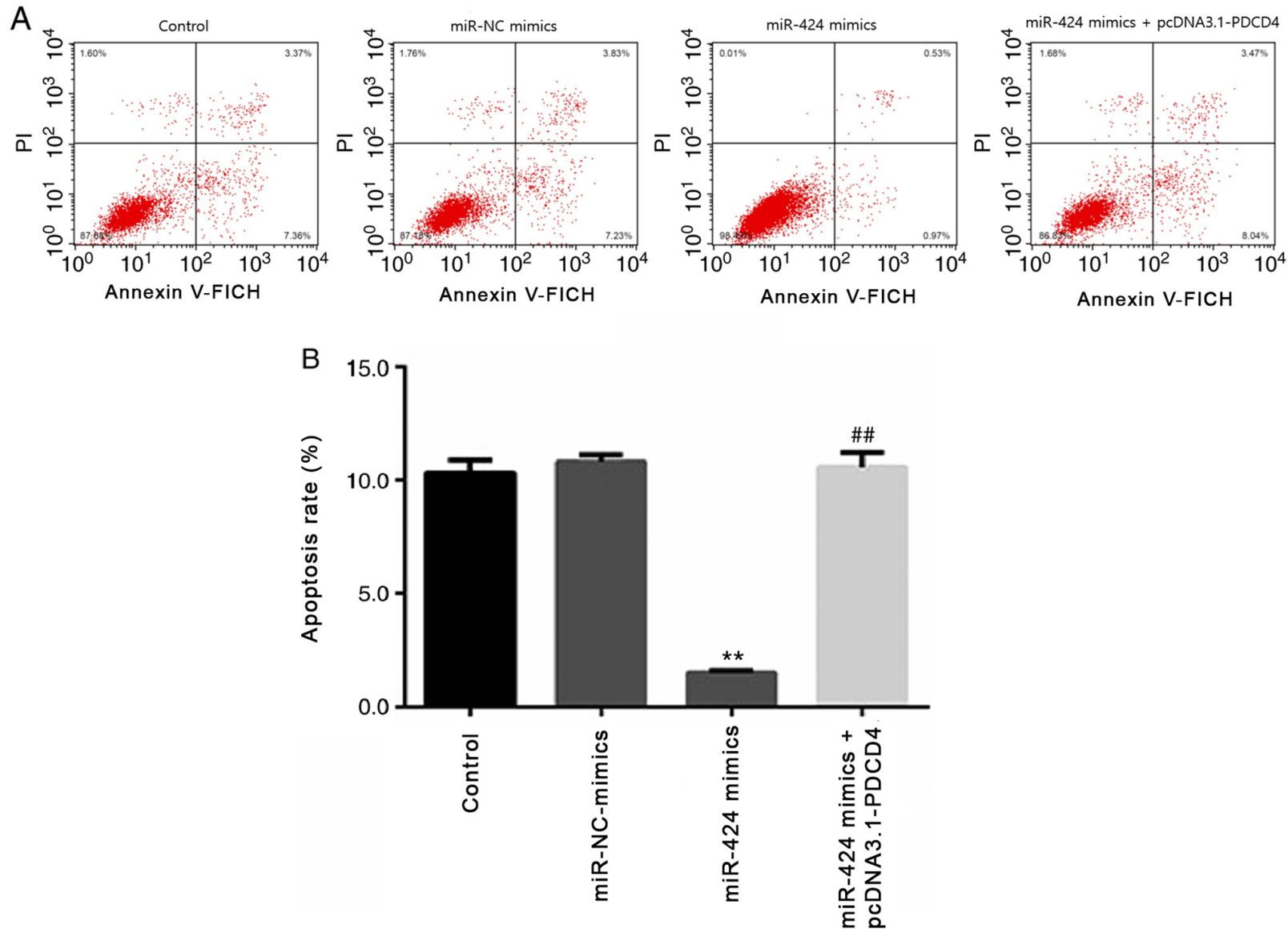

Figure 5. Overexpression of miR-424 inhibits the apoptosis of $\mathrm{CoCl}_{2}$-treated $\mathrm{PC} 12$ cells. (A) Overexpression of miR-424 significantly suppressed the apoptosis of $\mathrm{CoCl}_{2}$-treated PC12 cells compared with the miR-NC mimics group, which was significantly reversed after co-transfection with pcDNA3.1-PDCD4. There was no significant difference between the control and $\mathrm{NC}$ groups. (B) Quantification of flow cytometry assay. ${ }^{* *} \mathrm{P}<0.01 \mathrm{vs}$. miR-NC mimics; ${ }^{* \#} \mathrm{P}<0.01$ vs. miR-424 mimics. miR, microRNA; PDCD4, programmed cell death protein 4; NC, negative control; PI, propidium iodide.

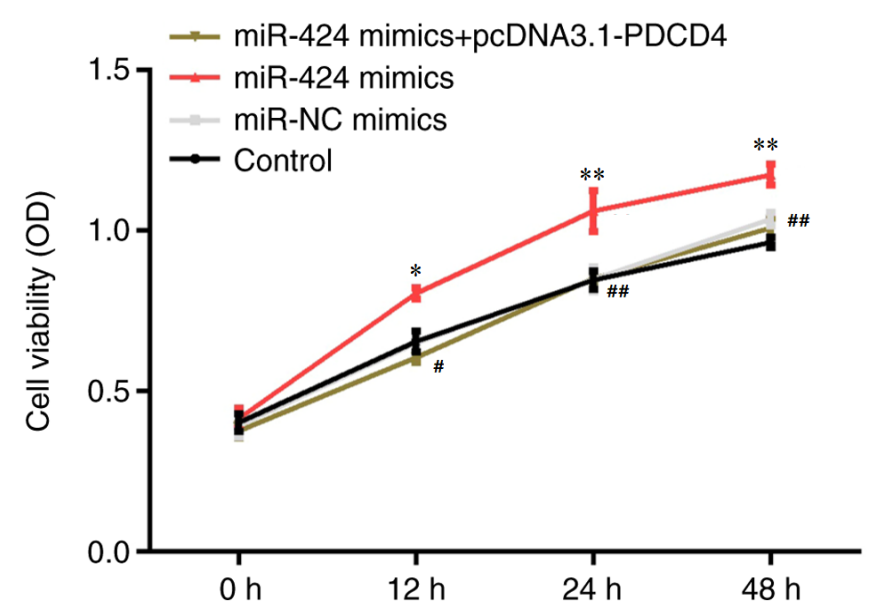

Figure 6. Overexpression of miR-424 promotes the viability of $\mathrm{CoCl}_{2}$-treated PC12 cells. Increased miR-424 expression promoted the viability of $\mathrm{CoCl}_{2}$-treated $\mathrm{PC} 12$ cells, which was significantly reversed after co-transfection with pcDNA3.1-PDCD4. There was no significant difference between the control and NC groups. "Between miR-424 mimics and miR-424 mimics+pcDNA3.1PDCD4. "Between miR-424 mimics and miR-NC mimics. ${ }^{*} \mathrm{P}<0.05,{ }^{* * *} \mathrm{P}<0.01$, ${ }^{\#} \mathrm{P}<0.05,{ }^{\# \prime} \mathrm{P}<0.01$ vs. miR-424 mimics. miR, microRNA; $\mathrm{PDCD} 4$, programmed cell death protein 4; NC, negative control; OD, optical density.

PC12 cells, which was reversed following co-transfection with pcDNA3.1-PDCD4 at 12, 24 and $48 \mathrm{~h}$ (Fig. 6)
miR-424 regulates the expression of PDCD4, Bcl-2, Bax, caspase-3, PI3K/AKT and phosphorylated (p)-PI3K/AKT. RT-qPCR and western blotting were conducted to evaluate mRNA and protein expression, respectively. As presented in Fig. 7A-D, the mRNA expression of PDCD4, Bax and caspase-3 was downregulated following miR-424 overexpression, whereas Bcl-2 expression was increased; these effects were significantly reversed by co-transfection with pcDNA3.1-PDCD4. There was no significant difference between the control and miR-NC mimics groups. Protein expression exhibited the same trends as mRNA expression. Furthermore, the levels of p-PI3K and p-AKT in cells transfected with miR-424 mimics were significantly increased, which was significantly reversed following co-transfection with pcDNA3.1-PDCD4. The protein levels of PI3K/AKT were not significantly affected by any transfections (Fig. 7E and F).

\section{Discussion}

Increasing evidence has indicated that aberrant expression of miRNAs in the human brain serves a crucial role in various brain diseases, including cerebral ischemic stroke $(31,32)$. In the present study, the roles of miR-424 in cerebral ischemic stroke were investigated. The results indicated that miR-424 was downregulated in $\mathrm{CoCl}_{2}$-treated $\mathrm{PC} 12$ cells. Overexpression of miR-424 increased cell viability and inhibited apoptosis in 
A

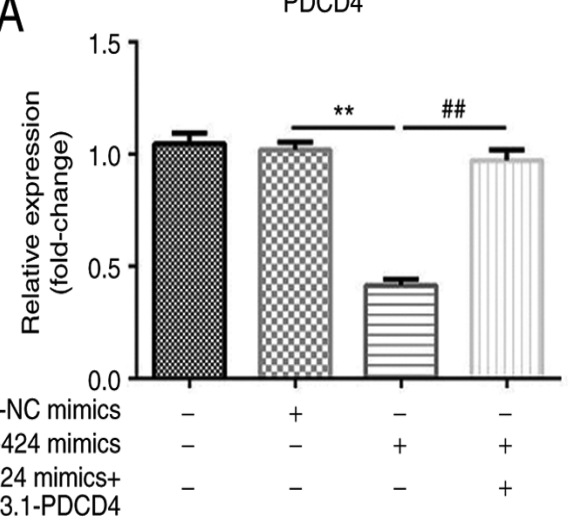

C

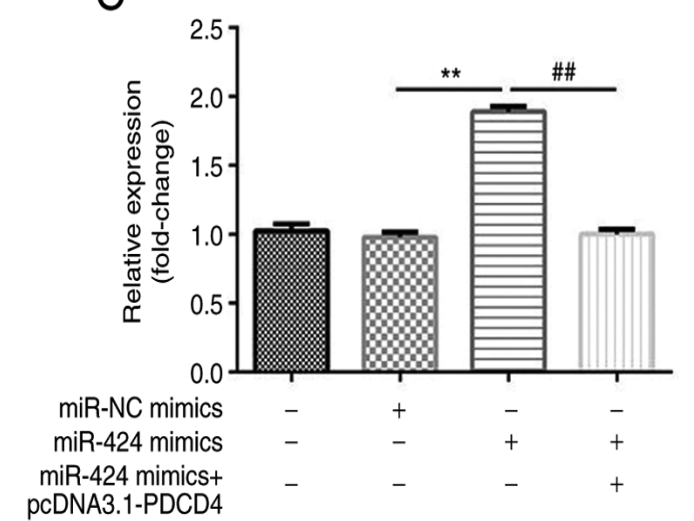

B

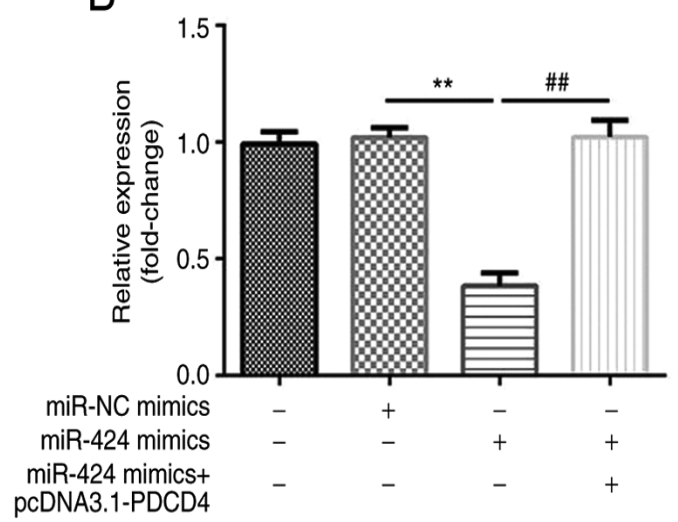

D

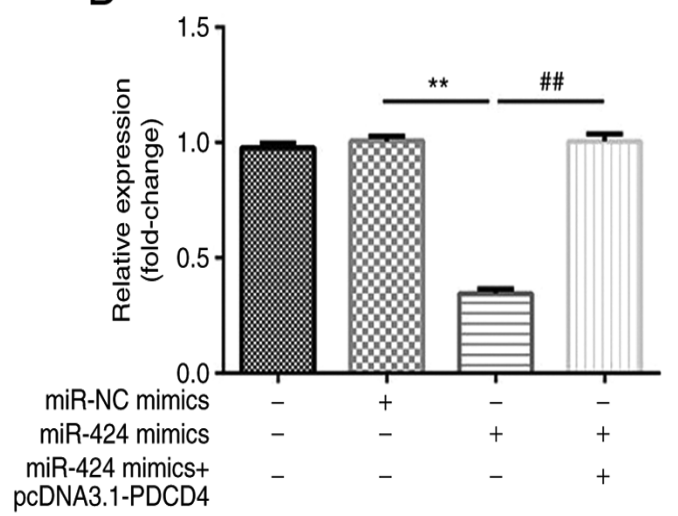

E

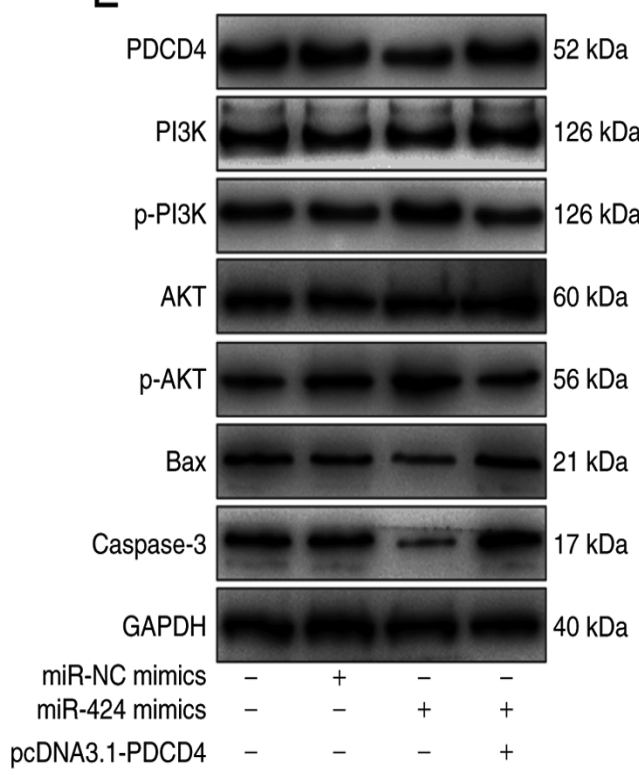

F
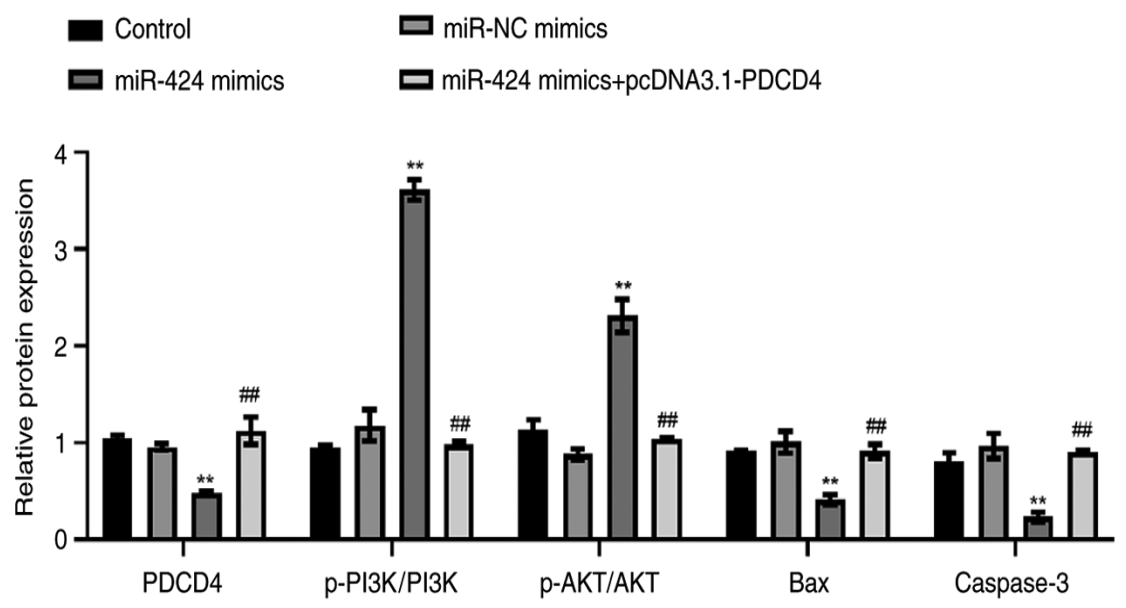

Figure 7. miR-424 regulates the expression of PDCD4, Bcl-2, Bax, caspase-3, PI3K/AKT and p-PI3K/AKT. Overexpression miR-424 altered the mRNA expression of (A) PDCD4, (B) Bax, (C) Bcl-2 and (D) caspase-3; its effects were attenuated by pcDNA3.1-PDCD4 co-transfection. (E) miR-424 overexpression increased the relative phosphorylation of PI3K and AKT, and suppressed PDCD4, Bax and caspase-3; its effects were attenuated by pcDNA3.1-PDCD4 co-transfection. (F) Qualitative analysis of western blotting. ${ }^{* *} \mathrm{P}<0.01 \mathrm{vs}$. miR-NC mimics or as indicated; ${ }^{\# \#} \mathrm{P}<0.01 \mathrm{vs}$. miR-424 mimics or as indicated. miR, microRNA; PDCD4, programmed cell death protein 4; NC, negative control; p, phosphorylated.

the ischemia model, which suggested that miR-424 may be a neuroprotective miRNA in cerebral ischemic stroke. This was consistent with a previous study that demonstrated that miR-424 mediated neuronal damage (33). However, the potential underlying mechanisms remain to be elucidated.
Neuronal cell death is considered to be the primary cause of stroke pathophysiology (34). This process can be slowed down by miRNAs via the regulation of apoptosis in neuronal cells (35). For example, overexpression of miR-455 exerted a positive role in protecting neuronal cells from 
ischemic injury-induced death (36). Notably, downregulation of miR-155 serves a protective role in ischemic stroke (37). In the present study, overexpression of miR-424 inhibited the apoptosis of neuronal cells. Furthermore, increased miR-424 promoted the viability of $\mathrm{CoCl}_{2}$-treated $\mathrm{PC} 12$ cells, suggesting that miR-424 may serve a neuroprotective role in cerebral ischemic stroke.

miRNAs regulate gene expression via binding sites in the 3'UTR of target genes (38). PDCD4 was predicted to be a target gene of miR-424. In the present study, overexpression of miR-424 decreased the expression of PDCD4, which was reversed after co-transfection with pcDNA3.1-PDCD4. Moreover, luciferase activity was decreased after co-transfection with miR-424 mimics and PDCD4-3'UTR-WT compared with miR-NC mimics, suggesting that PDCD4 is a target of miR-424. PDCD4 is an apoptotic protein and serves an important role in short-term responses to ischemic stroke (39). Additionally, associations between PDCD4 and miRNAs have previously been reported in cerebral ischemia (40). In the present study, co-transfection with PDCD4 attenuated the decrease in apoptotic rate induced by overexpression of miR-424, and inhibited cell viability. However, the underlying mechanisms remain unclear. The possible effects of PDCD4 on the expression of various genes and proteins were examined. It was revealed that miR-424 overexpression increased the expression of $\mathrm{Bcl}-2$ and the phosphorylation of PI3K and AKT, and decreased Bax and caspase- 3 expression; these effects were reversed by co-transfection with pcDNA3.1-PDCD4. Taken together, the present results indicated that miR-424 may regulate neuronal apoptosis by targeting PDCD4.

In conclusion, miR-424 was downregulated in ischemia-injured cells. miR-424 may serve a neuroprotective role by inhibiting the apoptosis and promoting the viability of ischemia-injured cells, potentially by targeting PDCD4. This may be a novel therapeutic target for cerebral ischemic stroke.

\section{Acknowledgements}

Not applicable.

\section{Funding}

The present study was supported by The Scientific Research Fund of Taizhou People's Hospital (grant no. ZL202015).

\section{Availability of data and materials}

The datasets used and/or analyzed during the current study are available from the corresponding author on reasonable request.

\section{Authors' contributions}

HWR performed the majority of experiments and wrote the manuscript. BG, YZZ, TG, QW and YQS performed the remaining experiments and statistically analyzed the data. JW designed the present study. HWR and JW can authenticate the raw data in this study. All authors read and approved the final manuscript.

\section{Ethics approval and consent to participate}

Not applicable.

\section{Patient consent for publication}

Not applicable.

\section{Competing interests}

The authors declare that they have no competing interests.

\section{References}

1. Johnston SC, Mendis S and Mathers CD: Global variation in stroke burden and mortality: Estimates from monitoring, surveillance, and modelling. Lancet Neurol 8: 345-354, 2009.

2. Zhao WJ, Zhang HF and Su JY: Downregulation of microRNA-195 promotes angiogenesis induced by cerebral infarction via targeting VEGFA. Mol Med Rep 16: 5434-5440, 2017.

3. Lo EH, Dalkara T and Moskowitz MA: Mechanisms, challenges and opportunities in stroke. Nat Rev Neurosci 4: 399-415, 2003.

4. Donnan GA, Fisher M, Macleod M and Davis SM: Stroke. Lancet 371: 1612-1623, 2008.

5. Xu G, Ma M, Liu X and Hankey GJ: Is there a stroke belt in China and why? Stroke 44: 1775-1783, 2013.

6. Kleindorfer D, de los Rios La Rosa F, Khatri P, Kissela B, Mackey $\mathrm{J}$ and Adeoye O: Temporal trends in acute stroke management. Stroke 44 (Suppl 1): S129-S131, 2013.

7. Vemuganti R: All's well that transcribes well: Non-coding RNAs and post-stroke brain damage. Neurochem Int 63: 438-449, 2013.

8. Truelsen T and Bonita R: Epidemiological transition of stroke in China? Stroke 39: 1653-1654, 2008.

9. Wang W, Jiang B, Sun H, Ru X, Sun D, Wang L, Wang L, Jiang Y, Li Y, Wang Y, et al; NESS-China Investigators: Prevalence, Incidence, and Mortality of Stroke in China: Results from a Nationwide Population-Based Survey of 480,687 Adults. Circulation 135: 759-771, 2017.

10. Xing B, Chen H, Zhang M, Zhao D, Jiang R, Liu X and Zhang S: Ischemic postconditioning inhibits apoptosis after focal cerebral ischemia/reperfusion injury in the rat. Stroke 39: 2362-2369, 2008.

11. Townsend PA, Scarabelli TM, Pasini E, Gitti G, Menegazzi M, Suzuki H, Knight RA, Latchman DS and Stephanou A: Epigallocatechin-3-gallate inhibits STAT-1 activation and protects cardiac myocytes from ischemia/reperfusion-induced apoptosis. FASEB J 18: 1621-1623, 2004.

12. Li P, Shen M, Gao F, Wu J, Zhang J, Teng F and Zhang C: An Antagomir to MicroRNA-106b-5p Ameliorates Cerebral Ischemia and Reperfusion Injury in Rats Via Inhibiting Apoptosis and Oxidative Stress. Mol Neurobiol 54: 2901-2921, 2017.

13. Pasquinelli AE: MicroRNAs and their targets: Recognition, regulation and an emerging reciprocal relationship. Nat Rev Genet 13: 271-282, 2012.

14. Ghelani HS, Rachchh MA and Gokani RH: MicroRNAs as newer therapeutic targets: A big hope from a tiny player. J Pharmacol Pharmacother 3: 217-227, 2012

15. Croce CM and Calin GA: miRNAs, cancer, and stem cell division. Cell 122: 6-7, 2005.

16. Zheng Y, Wang L, Chen M, Pei A, Xie L and Zhu S: Upregulation of miR-130b protects against cerebral ischemic injury by targeting water channel protein aquaporin 4 (AQP4). Am J Transl Res 9: 3452-3461, 2017.

17. Zheng L, Cheng W, Wang X, Yang Z, Zhou X and Pan C: Overexpression of MicroRNA-145 Ameliorates Astrocyte Injury by Targeting Aquaporin 4 in Cerebral Ischemic Stroke. BioMed Res Int 2017: 9530951, 2017.

18. Yin KJ, Hamblin M and Chen YE: Non-coding RNAs in cerebral endothelial pathophysiology: Emerging roles in stroke. Neurochem Int 77: 9-16, 2014.

19. Zhao L, Hua C, Li Y, Sun Q and Wu W: miR-525-5p inhibits ADAMTS13 and is correlated with Ischemia/reperfusion injury-induced neuronal cell death. Int J Clin Exp Med 8: 18115-18122, 2015 
20. Wei N, Xiao L, Xue R, Zhang D, Zhou J, Ren H, Guo S and Xu J: MicroRNA-9 Mediates the Cell Apoptosis by Targeting Bcl2111 in Ischemic Stroke. Mol Neurobiol 53: 6809-6817, 2016.

21. Zhang YZ, Wang J and Xu F: Circulating miR-29b and miR-424 as Prognostic Markers in Patients with Acute Cerebral Infarction. Clin Lab 63: 1667-1674, 2017.

22. Darbinian N: Cultured Cell Line Models of Neuronal Differentiation: NT2, PC12, and SK-N-MC. Methods Mol Biol 2311: 25-38, 2021.

23. Katarzyna B and Benita W: PC12 and THP-1 Cell Lines as Neuronal and Microglia Model in Neurobiological Research. Appl Sci (Basel) 11: 3729, 2021.

24. Kudo TA, Tominami K, Izumi S, Hayashi Y, Noguchi T, Matsuzawa A, Hong G and Nakai J: Characterization of PC12 Cell Subclones with Different Sensitivities to Programmed Thermal Stimulation. Int J Mol Sci 21: 8356, 2020.

25. Wiatrak $B$ and Balon K: Protective Activity of $A \beta$ on Cell Cultures (PC12 and THP-1 after Differentiation) Preincubated with Lipopolysaccharide (LPS). Mol Neurobiol 58: 1453-1464, 2021.

26. Amalia L, Sadeli HA, Parwati I, Rizal A and Panigoro R: Hypoxia-inducible factor- $1 \alpha$ in acute ischemic stroke: Neuroprotection for better clinical outcome. Heliyon 6: e04286, 2020.

27. Tripathi VK, Subramaniyan SA and Hwang I: Molecular and Cellular Response of Co-cultured Cells toward Cobalt Chloride $\left(\mathrm{CoCl}_{2}\right)$-Induced Hypoxia. ACS Omega 4: 20882-20893, 2019.

28. Kim HB, Yoo JY, Yoo SY, Lee JH, Chang W, Kim HS, Baik TK and Woo RS: Neuregulin-1 inhibits $\mathrm{CoCl}_{2}$-induced upregulation of excitatory amino acid carrier 1 expression and oxidative stress in SH-SY5Y cells and the hippocampus of mice. Mol Brain 13: $153,2020$.

29. Matsuhashi S, Manirujjaman M, Hamajima $\mathrm{H}$ and Ozaki I: Control mechanisms of the tumor suppressor PDCD4: Expression and functions. Int J Mol Sci 20: 2304, 2019.

30. Livak KJ and Schmittgen TD: Analysis of relative gene expression data using real-time quantitative PCR and the 2(-Delta Delta C(T)) Method. Methods 25: 402-408, 2001.

31. Saugstad JA: MicroRNAs as effectors of brain function. Stroke 44 (Suppl 1): S17-S19, 2013.
32. Yan H, Fang M and Liu XY: Role of microRNAs in stroke and poststroke depression. ScientificWorldJournal 2013: 459692, 2013.

33. Gui SH, Wang YM, Fan CY, Han LK, Wang TG, Di MQ and Gu JQ: miR-424 Inhibits neuronal apoptosis in rats with cerebral infarction through regulating TGF- $\beta 1 / \mathrm{Smad} 3$ signaling pathway. J Biol Regul Homeost Agents 34: 1285-1296, 2020.

34. Sun H, Zhong D, Wang C, Sun Y, Zhao J and Li G: MiR-298 Exacerbates Ischemia/Reperfusion Injury Following Ischemic Stroke by Targeting Act1. Cell Physiol Biochem 48: 528-539, 2018.

35. Baczyńska D, Michałowska D and Witkiewicz W: The role of microRNA in ischemic diseases - impact on the regulation of inflammatory, apoptosis and angiogenesis processes. Przegl Lek 70: 135-142, 2013 (In Polish).

36. Yao S, Tang B, Li G, Fan R and Cao F: miR-455 inhibits neuronal cell death by targeting TRAF3 in cerebral ischemic stroke. Neuropsychiatr Dis Treat 12: 3083-3092, 2016.

37. Xing G, Luo Z, Zhong C, Pan X and Xu X: Influence of miR-155 on Cell Apoptosis in Rats with Ischemic Stroke: Role of the Ras Homolog Enriched in Brain (Rheb)/mTOR Pathway. Med Sci Monit 22: 5141-5153, 2016.

38. Schwarzenbach H, Nishida N, Calin GA and Pantel K: Clinical relevance of circulating cell-free microRNAs in cancer. Nat Rev Clin Oncol 11: 145-156, 2014.

39. Guo YB, Ji TF, Zhou HW and Yu JL: Effects of microRNA-21 on Nerve Cell Regeneration and Neural Function Recovery in Diabetes Mellitus Combined with Cerebral Infarction Rats by Targeting PDCD4. Mol Neurobiol 55: 2494-2505, 2018.

40. Yan H, Rao J, Yuan J, Gao L, Huang W, Zhao L and Ren J: Long non-coding RNA MEG3 functions as a competing endogenous RNA to regulate ischemic neuronal death by targeting miR-21/PDCD4 signaling pathway. Cell Death Dis 8: 3211, 2017.

This work is licensed under a Creative Commons Attribution-NonCommercial-NoDerivatives 4.0 International (CC BY-NC-ND 4.0) License. 